\title{
Practical Advice: Navigating the Landscape for Using Current 3D Body Scanners in a Medical Environment. Do's, Don'ts and "Maybes"
}

\author{
David B. STEFAN ${ }^{1}$, David A. GILBERT ${ }^{2}$ \\ ${ }^{1}$ Novaptus Systems Inc., Chesapeake VA, USA; \\ ${ }^{2}$ The Hague Plastic and Cosmetic Surgery Center, Norfolk VA, USA \\ DOI: $10.15221 / 16.111$ http://dx.doi.org/10.15221/16.111
}

\section{Background}

The medical branch of sciences can appear like a close-knit, difficult fraternity to enter. This in part is due to sensitivities about historical incidents that today, would border on what would be called "quackery," or "charlatanism." In the name of both protecting the profession as well as the patient, the equivalent of trade associations were created. These self-regulating associations established early standards for the medical profession. Later, governmental agencies developed and superseded private organizations as regulatory authorities. Today, private associations and public government agencies combine in such a way as to as to form what looks to many as an insular community. This "establishment" is a double-edged sword. On one hand, it has served its purpose by elevating the bar for quality of practitioners and consistency of care. On the other hand, the myriad of rules and regulations make it difficult for new ideas and new applications to enter from the outside. Worse, these rules have a tendency to change and can be subject to different interpretations. This paper gives a primer, based on practical experience, on how to field a 3D scanner system into a medical facility for research purposes. It also outlines a path to pre-market approval and commercialization for use within the medical arena.

\section{Introduction}

There is a threshold that must be crossed if you wish to take a commercially available product commonly in use outside the medical community and transplant it within a clinical environment. This is true even if the use of the product in the medical arena is not materially different from its use in other commercial trades.

Such is the case with a common 3D booth-type body scanner. These 3D body scanners are widely used in the clothing industry for measurement purposes, either for custom tailored clothing or for population sizing studies. Many of these sizing studies are sponsored by various government agencies.

There are rules and regulations that govern use of any medications, topical or ingested, or equipment, including tools and devices, that are used on human subjects. In part, this is because of past transgressions in the area of medical research that had tragic consequences. This led to the question of how to address, from an ethical standpoint, whether future research to be conducted has social merit, and how such research on humans can be performed.

\section{Brief History of Medical Research Ethics ${ }^{1}$}

Prior to the twentieth century, research ethics were primarily governed by individual conscience and professional codes of conduct. Historically, these have always been subject to the laws and customs of a particular society and/or government at the time. In the mid-twentieth century, the culmination of past events led to a concerted effort to establish a set of firm rules and regulations about research on human subjects in order to protect individual, community and public interests.

Restrictions on research involving humans is not a recent phenomenon. There is evidence of physician guidelines in ancient Egypt where priests carried out much of the functions of the modern day general practitioner, and in the Old Testament of the Bible, particularly in Leviticus. The Code of Hammurabi $\left(18^{\text {th }}\right.$ century B.C.) gave detailed and explicit penalties for what we would call today medical malpractice. In many physician's offices today, one can usually find a copy of the Hippocratic Oath, attributed to the $5^{\text {th }}$ century B.C. physician. 
Sometimes the Hippocratic Oath is boiled down to the phrase "Do no harm." The pertinent phrase in the translation often quoted with regard to medical ethics is "...to abstain from all intentional wrongdoing and harm, especially from abusing the bodies of man or woman, bound or free."

\section{The Scientific Revolution and Medical Experimentation ${ }^{2}$}

In the late 1600's a renaissance in science surfaced. Methods of investigation, such as controlled observation, accurate reporting of results and independent validation took the place of haphazard experimentation, occultism and mysticism. These processes were, of course, applied to experiments involving humans but the number of individuals were generally few and sometimes included the researchers themselves.

The discovery that germs caused illness, in the late 1800's opened up more areas of research on human subjects. Huge strides were made in immunization against certain diseases. But there were some spectacular and tragic failures as well. A particularly notorious experiment by a Prussian professor of dermatology is of note. He conducted experiments aimed at immunizing healthy subjects against syphilis, in the 1890's. Serum from patients with syphilis was used to inoculate healthy children and adolescent prostitutes. This resulted in all subjects contracting the disease. No consent had been obtained from the subjects, nor had any permission been given by the parents or guardians.

The "outrage," fueled by press coverage prompted the Prussian government to issue "Instructions to the Directors of Clinics, Out-patient Clinics and Other Medical Facilities." It was one of the first known government edicts to promulgate what we now call "Informed Consent." Thus ethical problems began to come into question by public opinion, and ideas began to revolve around the notion of risk versus benefit of medical experimentation on human subjects.

\section{World War II and the Nuremberg Code}

After the conclusion of World War II, over 20 German physicians and administrators were brought to trial for their willing participation in "war crimes" and "crimes against humanity." Many medical experiments, on possibly several thousand concentration camp prisoners took place, without consent. These crimes were documented as part of the military tribunal that began in late 1946. At the conclusion of the tribunal in the middle of 1947, seven defendants were found guilty and sentenced to death, eight defendants were sentenced to prison for at least ten years and the rest were found not guilty.

Two American doctors who had worked on the prosecution team submitted a memorandum that outlined guidelines for "legitimate research" came to be known as The Nuremberg Code.

Among the points emphasized was the quality of the experiments and the competency of the researchers. Above all, was the requirement that the "voluntary consent of the human subject is absolutely essential."

The Nuremberg Code left the issue of compliance up to the investigator, which also left the door open for potentially unscrupulous behavior, and it had no enforcement mechanism should any malicious behavior result in unintended outcomes.

\section{Thalidomide Tragedy ${ }^{3}$}

The misuse of Thalidomide and its tragic results in the late 1950s was perhaps the turning point that forced the legislation which provided the framework for compliance and enforcement mechanisms. Thalidomide was approved as a sedative in Europe, but not in the United States. It was originally used to help sleep and reduce nausea associated with pregnancy. However, ingestion of this drug during pregnancy caused severe fetus deformities. Many who took the drug did not know that it was experimental, and therefore not approved for use, nor did they give their consent.

Several thousand infants were born with severe deformities.

This prompted a hearing in the U.S. Senate. In the early 1960's amendments to the Food, Drug and Cosmetic Act were enacted to ensure drug efficacy and drug safety. This episode marked the beginning of many of the FDA regulations that are in existence to this day. These regulations were centered around drugs for human consumption, requiring drug manufactures to prove to the FDA the effectiveness of their products before marketing them. 
Following this momentum, the World Medical Association issued guidelines for medical doctors in biomedical research involving human subjects. This established the precept that research with human subjects is justified only when the degree of risk to subjects does not exceed the humanitarian importance of the knowledge to be gained.

There were other papers published in in mid-1960's arguing that some medical research that was being conducted was 'thoughtless and careless" and had little therapeutic value to patients or medicine. These caught the attention of the media and inflamed American public opinion.

\section{The Introduction of Independent Review Boards (IRB)}

To placate an increasingly wary public, in 1965, the predecessor to the National institutes of Health in the U.S. announced that it would publish guidelines requiring approval of protocols for research on human subjects before a study could begin. Such a memorandum was published by the Research Grants Division in February of 1966.

Highlights of the new policy were: Involvement of an Institution; Safeguarding of Individual Rights, Involvement of a committee of "outside associates" for independent judgement; Mandatory informed consent; Determination of risks versus benefits.

In essence, this memorandum mandated use of Institutional Review Boards that were made up of qualified outside reviewers.

Finally, as an enforcement mechanism, compliance of the new rules was directly tied to funding approval or renewal if the funds came from a government agency.

\section{The Tuskegee Syphilis Study $(1932-1972)^{4}$}

There was another episode of note that occurred after the publication of the 1966 memorandum establishing Institutional Review Boards for research involving human subjects.

This was the infamous Tuskegee Syphilis Study, which occurred between 1932 and 1972, conducted by the same organization that issued the new research guidelines.

Six-hundred low income African-American males from rural Alabama with a high incidence of syphilis infection were monitored for 40 years. These subjects were given free medical examinations, but they were not told about their disease. Even though a proven cure (penicillin) became available in the 1950s, the study continued until 1972 with participants and their families being denied treatment.

The study was stopped in 1973 by what had become by then the U.S. Department of Health, Education and Welfare only after it was exposed in a newspaper story. The public outrage became a political fiasco. This heightened awareness of the need to protect human subjects and assure their informed voluntary consent to participate in research on human subjects.

Due to the publicity of the Tuskegee Syphilis Study, the National Research Act of 1974 was passed. This created a commission that was charged to identify the basic ethical principles that should underlie the conduct of both biomedical and behavioral research involving human subjects.

\section{The Belmont Report ${ }^{5}$}

The Commission produced the Belmont Report, published in 1979, which set the foundation for the ethics of research involving human subjects in the United States.

Three principle ideas were established. They were "Respect for persons," "Beneficence," and "Justice."

The main point under "Respect for persons" is "Informed Consent." That subjects, to the degree that they are capable, must be given the opportunity to choose what shall or what shall not happen to them, and that the consent process must include three elements. These three elements are information, comprehension, and voluntariness.

Under "Beneficence" comes the assessment of risks and benefits. That is, the nature and scope of the risks and benefits must be assessed in a systematic manner.

Finally, under "Justice" come the precept that the benefits and risks of research must be distributed fairly. 


\section{The Common Rule (Present Day Guidelines)}

In 1991, the various differences between government organizations that have a hand in regulating research on human subjects published what was known as the "Common Rule."

The main elements of the Common Rule include:

- requirements for assuring compliance by research institutions;

- requirements for researchers obtaining and documenting informed consent;

- requirements for Institutional Review Board (IRB) membership, function, operations, review of research, and record keeping.

- additional protections for certain vulnerable research subjects-- pregnant women, prisoners, and children

In addition, certain federally sponsored and much privately sponsored research is subject to the regulations of the Food and Drug Administration (FDA) at 21 CFR Parts 50 and 56. FDA regulations confer protections on human subjects in research when a drug, device, biologic, food additive, color additive, electronic product, or other test article subject to FDA regulation is involved. FDA regulations and the provisions of the Common Rule are largely congruent, although some significant differences exist.

Both the Common Rule and the FDA regulations provide protections for human subjects in research.

\section{Summary of the History of Medical Research Ethics}

So as one can readily see, the results of "wayward" past medical experimentation on human subjects arose public outrage and led to the creation of Institutional Review Boards to not only oversee medical research, but also to make a judgement as to the merit of such research. Deeply intertwined within the function of the IRB is the notion of "Informed Consent" on the part of the human subject.

We have also seen that since the mid-1960's an enormous amount of medical research funds started to be provided by government sources in the form of research grants. Compliance to the rules and regulations that were developed in order to maintain or receive funding was the effective enforcement mechanism.

Many of the abuses documented in the above history centered around internal medicine, either in the ingestion of unapproved drugs, or injection of unapproved vaccines in search of immunization against certain diseases, or even denial of approved drugs to cure diagnosed diseases.

The rules, regulations and enforcement are a formidable combination, developed over time to justifiably prevent past documented abuses in medical research ethics from recurring. To anyone trying to place commercially available equipment from outside the medical community into the medical environment for research purposes, it might appear that these rules and regulations are simply too complicated and therefore insurmountable. But that isn't necessarily the case.

\section{The Definitions of a Medical Device and the Role of the FDA}

There have been many opinions as to what constitutes a medical device. To understand why this statement includes the word "opinion," one need only to go to the website of the authority for medical devices, the United States Food and Drug Administration.

The following is taken directly from the FDA website, the font is different to maintain separation of what was taken from the website, and the text of this paper: 


\section{"Medical Devices: The Basics"}

The definition has several components. A medical device:

- diagnoses, cures, lessens, treats, or prevents disease

- affects the function or structure of the body

- does not achieve primary intended purposes through chemical action

FDA's Center for Devices and Radiological Health regulates companies that design, manufacture, repackage, relabel, and/or import medical devices into the United States. The agency does not regulate the practice of medicine - how and which physicians can use a device. The only exception is FDA's regulation of mammography facilities under the Mammography Quality Standards Act.

\section{Medical Device Law}

The long legal journey toward medical device regulation began with the Pure Food and Drugs act of 1906. Medical devices were not included as no one envisioned how technology would grow increasingly complex and need to be regulated. The Medical Device Amendments of 1976 gave FDA authority to ensure the safety and effectiveness of a range of life-saving medical devices while also protecting the public from fraudulent devices. The Amendments:

- defined a medical device,

- established three device classes (I, II, and III),

- identified pathways to market,

- established Advisory Panels, and

- set clinical investigation requirements.

Subsequent legislation strengthened the FDA's regulatory authority:

\section{Three classes of regulatory control}

The three device classes are based on the degree of regulatory control necessary to ensure their safety and effectiveness:

Class I devices present a low risk of harm to the user and are subject to general controls that are sufficient to protect the user. Most are exempt from the regulatory process.

Examples: non-powered breast pumps, elastic bandages, tongue depressors, examination gloves, most hearing aids, arm slings, microbial analyzers, keratoscopes.

Class II devices are more complicated and require special controls for labeling, guidance, tracking, design, performance standards, and post market monitoring. Most require Premarket Notification 510(k). (Emphasis highlighted by author)

Examples: powered wheelchairs, CT scanners, contact lens care products, endolymphatic shunts

Class III devices usually sustain or support life, are implanted, or present potential unreasonable risk of illness or injury. They have the toughest regulatory controls. Most of these devices require Premarket Approval because general and special controls alone cannot reasonably assure their safety and effectiveness.

Examples: pacemakers, implanted weight loss devices, non-invasive glucose testing devices, medical imaging analyzers, cochlear implants, breast implants."

There is a difference between medical research using a device and the commercialization of that particular device into the medical market. The path to the marketplace can be different than the path to prove the technology during the research phase.

This is a very important point, particular as the technology for 3D body scanning advances and improves.

The following continues from the pertinent section of the FDA website: 


\section{How FDA Reviews Medical Devices.}

\section{Investigational Device Exemptions (IDE)}

An IDE allows an investigational device to be used in a clinical study to collect the safety and effectiveness data required for a Premarket Approval (PMA) application or a Premarket Notification (510(k)) submission to FDA. Clinical studies with devices of significant risk must be approved by both FDA and an Institutional Review Board (IRB) before the study can begin. Studies with devices posing non-significant risk must be approved by an IRB before the study can begin (emphasis added by the author).

FDA observes a 30-day review period for IDE applications. The agency focuses its review on the data provided to demonstrate the safety and anticipated benefits of the device for use in humans, as well as the scientific validity of the proposed clinical trial protocol.

Following clinical studies, a device's journey to market can take one of three major pathways:

1. Investigational Device Exemptions (IDE)

2. Premarket Notification $(510(\mathrm{k}))$

3. Premarket Approval Application (PMA)

4. Humanitarian Device Exemption (HDE)

\section{Premarket Notification $(510(k))$}

$510(\mathrm{k})$ is required when demonstrating substantial equivalence to a legally marketed device, when making significant modifications to a marketed device, and when a person required to register with FDA introduces a device for the first time. If a device requires the submission of a 510(k), it cannot be commercially distributed until the FDA authorizes it.

\section{Substantial Equivalence}

A device is substantially equivalent (SE) if it has the same intended use and same technological characteristics as a legally marketed device, known as the predicate. A legally marketed device:

1. was legally marketed prior to May 28, 1976 ("pre-amendments device"), for which a PMA is not required, or

2. was reclassified from Class III to Class II or Class I, or

3. was found SE through the $510(\mathrm{k})$ process.

Applicants must compare their device to one or more similar legally marketed devices and make and support their SE claims. If the device is SE to a predicate, it is placed in the same class. If it is not SE, it becomes non-SE and is placed into Class III.

Examples of 510(k)s include x-ray machines, dialysis machines, fetal monitors, lithotripsy machines, and muscle stimulators.

\section{What constitutes an "Electronic Product" and "Electromagnetic Radiation" as defined by the FDA?}

In general, all electronic devices that are used in the medical environment are subject to regulation by the FDA. This is a very broad statement, as all electronic devices emit some sort of radiation by their very nature.

Below is a section of text that represents the codification of regulations as defined by Title 21 , Volume 8. Subpart A refers to the definition of "Electromagnetic radiation," and "Electronic product." 
[Code of Federal Regulations]

[Title 21, Volume 8]

[Revised as of April 1, 2015]

[CITE: 21CFR1000]

TITLE 21--FOOD AND DRUGS

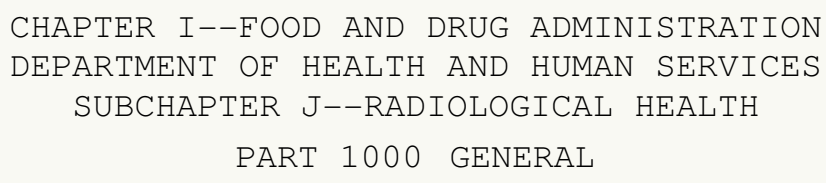

Subpart A--General Provisions

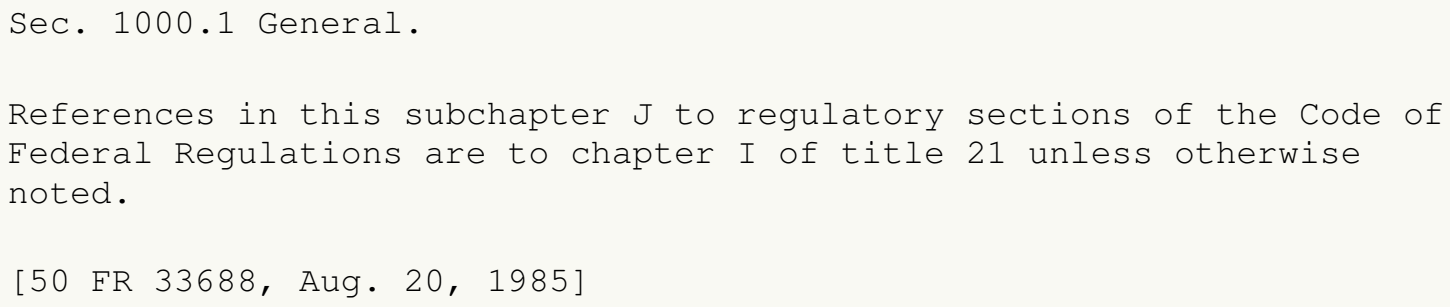

(i) Electromagnetic radiation includes the entire electromagnetic spectrum of radiation of any wavelength. The electromagnetic spectrum illustrated in figure 1 includes, but is not limited to, gamma rays, x-rays, ultra-violet, visible, infrared, microwave, radiowave, and low frequency radiation.

(j) Electronic product means:

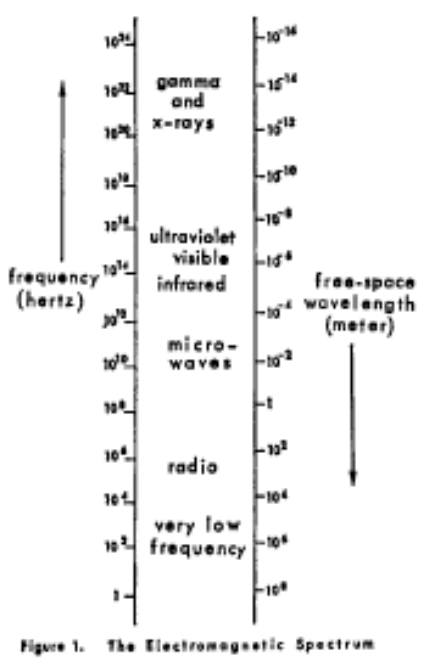

(1) Any manufactured or assembled product which, when in operation:

(i) Contains or acts as part of an electronic circuit and

(ii) Emits (or in the absence of effective shielding or other controls would emit) electronic product radiation, or

(2) Any manufactured or assembled article that is intended for use as a component, part, or accessory of a product described in paragraph (j)(1) of this section and which, when in operation, emits (or in the absence of effective shielding or other controls would emit) such radiation.

One can see that the entire electromagnetic spectrum is included. 
Subpart B of this regulation goes on to further define the combination "Electronic product radiation. ${ }^{8 \prime \prime}$

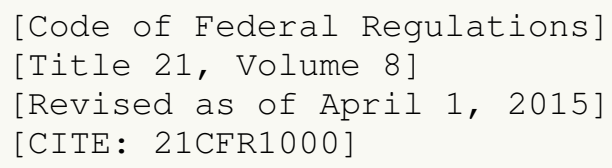

(k) Electronic product radiation means:

(1) Any ionizing or nonionizing electromagnetic or particulate radiation, or

(2) Any sonic, infrasonic, or ultrasonic wave that is emitted from an electronic product as the result of the operation of an electronic circuit in such product.

(1) Federal standard means a performance standard issued pursuant to section 534 of the Federal Food, Drug, and Cosmetic Act.

(m) Infrasonic, sonic (or audible) and ultrasonic waves refer to energy transmitted as an alteration (pressure, particle displacement or density) in a property of an elastic medium (gas, liquid or solid) that can be detected by an instrument or listener.

(b) Examples of electronic products which may emit ultraviolet, visible, infrared, microwaves, radio and low frequency electromagnetic radiation include:

Ultraviolet:

Biochemical and medical analyzers.

Tanning and therapeutic lamps.

Sanitizing and sterilizing devices.

Black light sources.

Welding equipment.

Visible: (Highlighted by the author)

White light devices.

\section{Infrared:}

Alarm systems.

Diathermy units.

Dryers, ovens, and heaters. 
So the reader can readily see that white light devices and examples of infrared devices are included within "Electronic Product Radiation," as listed is Subchapter J - "Radiological Health." The list isn't meant to be exhaustive, but the blanket coverage is intentional.

This leads to the question: How does one get a 3D body scanner (booth type or otherwise) into a clinical medical environment when the guidelines appear to be so restrictive?

Although the guidelines as written appear to make anything that emits electronic product radiation to be subject to the FDA's Center for Devices and Radiological Health approval, and rightly so, there are avenues available as long as one can assure that the device poses a "non-significant risk" to human subjects.

This is where the IRB can be very helpful.

The FDA does not want to inhibit innovation, it seeks only to provide protection for humans, and to make sure that any device used in a medical environment is fit for the application and does not pose any undue risk.

\section{A closer look at Investigational Device Exemptions}

The following are highlights taken from a presentation given by the FDA, it is available on their website at the following link?

http://www.fda.gov/downloads/medicaldevices/newsevents/workshopsconferences/ucm499250.pdf

Introduction to the Investigational
Device Exemption (IDE)
Regulations
Elaine Blyskun
OIR/DPOM

This presentation outlines the FDA's current thinking about Investigation Device Exemptions. It is often revised and updated, as the personnel change, but the overall flow charts have remained the same.

There are more detailed guidelines that amplify and substantiate what is discussed in this presentation. These details within the guidelines are constantly being revised, and some of the recommendations that are published specifically declare that they are non-binding ${ }^{10}$.

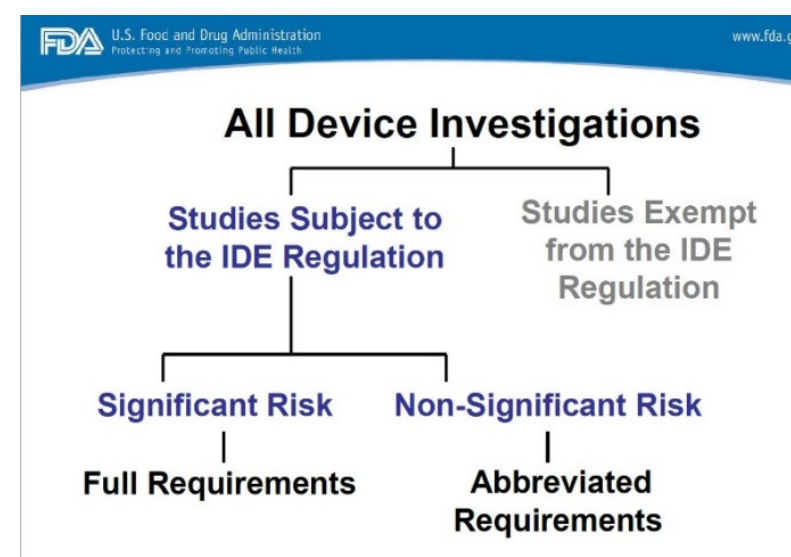


The 3D body scanner, of any kind, must be considered an electronic device, and ultimately is subject to Investigational Device Exemption approval. Not necessarily in its present form, but in the eventual form it will take when preparations are made to take it to market.

However, there are two paths under "Studies Subject to the IDE Regulation" heading. These are divided into two categories, "Significant Risk" and "Non-Significant Risk."

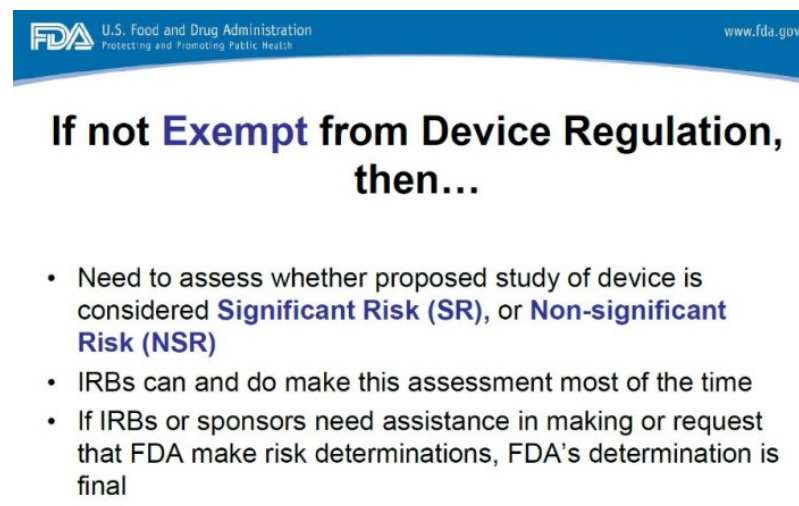

The key is for the IRB to make the determination as to whether the proposed study using the 3D scanner is considered Significant Risk, or Non-significant risk when used on human subjects.

What constitutes a device as having significant risk?

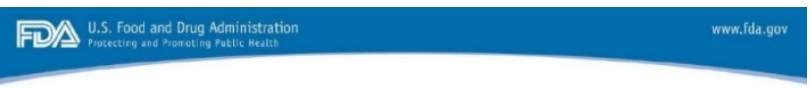

\section{Significant Risk Study}

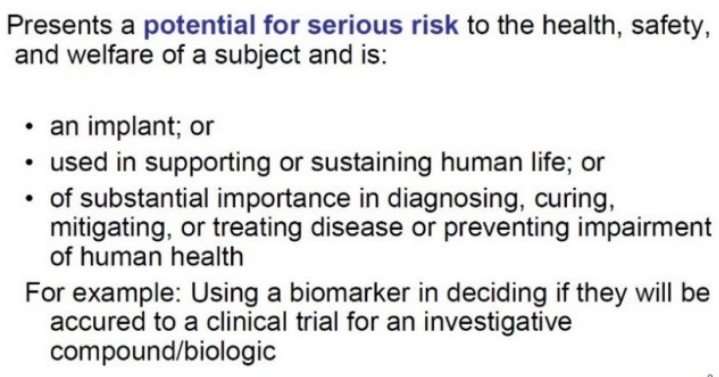

A 3D body scanner does not appear to be under any of these categories. It is not implantable. It is not used in supporting or sustaining human life. And as of yet, it is not of substantial importance in either diagnosing, curing, mitigating or treating a disease nor can it be used in preventing impairment of human health.
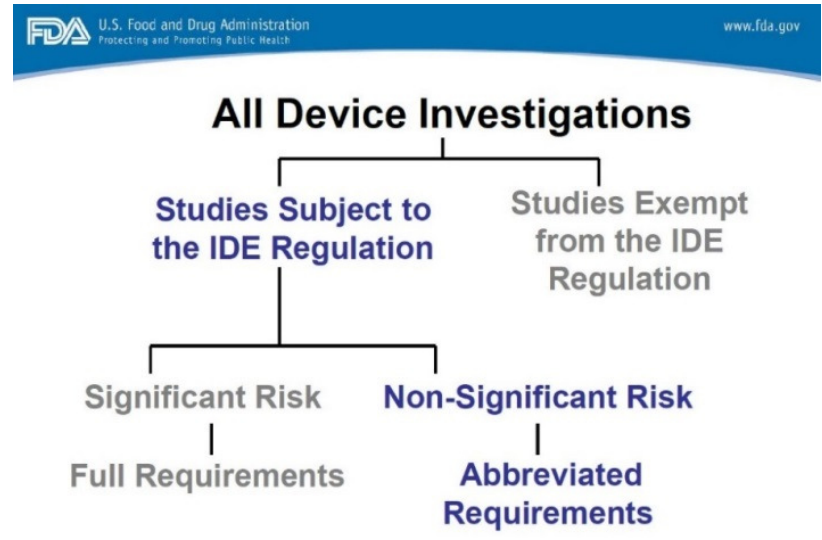
The decision tree path leads to the "Non-Significant Risk" branch, and within the "Non-Significant Risk" branch there are abbreviated requirements.

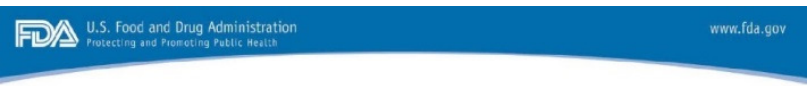

\section{Non-Significant Risk Studies}

- Sponsor presents protocol to IRB and a statement why the study does not pose a significant risk to the study participants

- If IRB approves the investigation as NSR, the sponsor may begin the study

- Abbreviated IDE requirements (labeling, IRB, informed consent, monitoring, reporting, prohibition of promotional activities)

- No IDE submission to FDA needed

For Devices NOT Currently Approved for General Marketing by the U.S. Food and Drug Administration (FDA): $:^{11}$

\section{Exceptions to the general requirements for an IDE submission}

- Clinical research studies involving the use of an unapproved device may be exempt from the requirement for an IDE submission if the research is not being conducted for the purpose of determining the safety and effectiveness of the device and the research use of the unapproved device does not put the subjects at significant risk.

- Clinical research studies of diagnostic devices are exempt from the IDE regulations if the testing:

1. is non-invasive*;

2. does not require an invasive sampling procedure that presents significant risk;

3. does not by design or intention introduce energy into a subject; and

4. is not used as a diagnostic procedure without confirmation by another medically established diagnostic product or procedure.

*The FDA defines 21 CFR 812.3 (k)) "noninvasive", when applied to a diagnostic device or procedure, as "one that does not by design or intention: (1) penetrate or pierce the skin or mucous membranes of the body, the ocular cavity, or the urethra; or (2) enter the ear beyond the external auditory canal, the nose beyond the nares, the mouth beyond the pharynx, the anal canal beyond the rectum, or the vagina beyond the cervical os." Blood sampling that involves simple venipuncture is considered noninvasive, and the use of surplus samples of body fluids or tissues that are left over from samples taken for non-investigational purposes are also considered noninvasive.

- If the use of the unapproved device in the research study involves greater than a minimal risk, the investigator is advised to seek clarification of the IDE submission from the FDA's Center for Devices and Radiological Health.

\section{The path to be taken that allows one to place a 3D body scanner into a medical environment}

This path requires a sponsor, that is, a principal investigator to conduct the research and design the protocol. The protocol must declare what it is that is under investigation, the benefits and risks of such research and restrictions on the population that will be part of the study (adults, juveniles, etc.).

The IRB committee must make a determination that the device used in the investigation is of a Nonsignificant risk (NSR) nature. They do this by evaluating the scanner's usage outside of the medical environment. In the 3D body scanner's case, it has been used quite often not only for custom tailored clothes, but also for numerous sizing studies for the clothing industry. It has also been used extensively for the development of standardized mannequins. Thus there is an assumption of 
"Substantial Equivalence," that is, the use of the 3D scanner in the medical environment is not substantially different than its use in the commercial environment.

Importantly, the 3D body scanner is used "as is." No modifications of the software or hardware is required for its use in the medical environment. This is an interesting point. The 3D scanner produces a $3 \mathrm{D}$ body model of the individual in the scanner. The measurement software allows one to program measurements as to how the body model is measured. Multiple measurement profiles can be used to measure the same body model, and in practice they often are employed. The 3D body model created by the scanner may even be exported to an "outside" program to perform the measurement extractions. Yet the function of the 3D body scanner remains unchanged. Its function is to produce an accurate 3D body model.

As part of the protocol submission process, a Subject Consent Form must be submitted for approval.

Once the IRB has determined that the device is "NSR," and approves the study protocol, there are still important points that one needs to keep in mind.

First, it is very important to inform the subject about the research and have them sign the consent form. This can't be done in a haphazard way. A separate consent form is needed, apart from other consent forms that the patient may be asked to read and sign.

Often, the patient is asked to read and sign many forms. It can be helpful for someone in the clinical office to administer and explain the forms, particularly the consent forms. Proper explanation of the consent form, in this case, for performing $3 \mathrm{D}$ body scanning can alleviate many if not all the concerns the individual may have. There is also the option not to participate in the study and therefore not get scanned.

The other functions of the IRB, once the device is considered "NSR" and the study is approved and underway is to perform a review of the study's progress. This is generally done on an annual basis unless there is some change to the study which needs to be addressed. Such changes can include turnover of the Principal Investigator, changes or modifications to the equipment (such as an updated scanner model) or a change in scope that alters the objectives of the study. Any of these changes require a written request to be submitted to the IRB, and the IRB, at its discretion can hold an "expedited" review to approve or reject any modifications.

Below is an image of the front page of an "IRB Continuing Review Report" which is required on an annual basis. Note that any changes to the existing protocol must be submitted using a separate Amendment Assessment form. There is an annual fee which varies from institution to institution.

This identifies the Study Title, the Principal Investigator and the Research Team Members, and where the research will be performed.

There are several pages to this review, and the original protocol may be many pages more and contain references supporting the research objectives. 
CONTINUING REVIEW REPORT

Amendments MUST be submitted separately using the Amendment Assessment form.

HELP: If you are unsure how to complete a field, press $\mathrm{F} 1$ while on the field and a help box will appear.

\begin{tabular}{|c|c|c|c|}
\hline & & \multirow{2}{*}{\multicolumn{2}{|c|}{ 05-09-FB-0235 }} \\
\hline & & & \\
\hline \multicolumn{4}{|c|}{ ADMINISTRATIVE INFORMATION } \\
\hline Study Title: & \multicolumn{2}{|l|}{ White Light Scanning to Aid Body Contouring Surgery } & \multirow[t]{3}{*}{ Date Submitted: (IRB USE ONLY) } \\
\hline Principal Investigator: & \multicolumn{2}{|l|}{ David A Gilbert, MD } & \\
\hline PI Dept / Address & \multicolumn{2}{|l|}{$\begin{array}{l}400 \text { W. Brambleton Ave. } \\
\text { Ste. } 300\end{array}$} & \\
\hline City / State / Zip & \multicolumn{3}{|l|}{ Norfolk, VA 23510} \\
\hline Phone Number(s): & $(757) 274-4000$ & E-Mail: & Dagib3@msn.com \\
\hline \multicolumn{4}{|c|}{ Person Preparing This Submission } \\
\hline Name: & David A Gilbert, MD & Role: & Investigator \\
\hline Address: & \multicolumn{3}{|l|}{$\begin{array}{l}400 \text { W. Brambleton Ave. } \\
\text { Ste. } 300 \\
\text { Norfolk, VA } 23510\end{array}$} \\
\hline Phone Number(s): & (757) $274-4000$ & E-Mail: & Dagib3@msn.com \\
\hline
\end{tabular}

\begin{tabular}{|c|c|c|c|c|}
\hline \multicolumn{5}{|c|}{ INVESTIGATORS ANDIOR RESEARCH TEAM MEMBERS } \\
\hline Name & Department & Address & Status & $\begin{array}{l}\text { Shince original } \\
\text { application } \\
\text { individual was: }\end{array}$ \\
\hline David A. Gilbert, MD & $\begin{array}{l}\text { Surgery (Plastic } \\
\text { Surgery) }\end{array}$ & $\begin{array}{l}400 \text { W. Brambleton Ave. } \\
\text { Ste. } 300 \\
\text { Norfolk, VA } 23510\end{array}$ & Research Team Member & On Original \\
\hline \multirow[t]{11}{*}{$\begin{array}{l}\text { Robert H.Schnarrs, } \\
\text { MD }\end{array}$} & $\begin{array}{l}\text { Surgery (Plastic } \\
\text { Surgery) }\end{array}$ & $\begin{array}{l}400 \text { W. Brambleton Ave. } \\
\text { Ste. } 300 \\
\text { Norfolk, VA } 23510\end{array}$ & Research Team Member & On Original \\
\hline & & & - Choose One - & - Choose One - \\
\hline & & & - Choose One - & - Choose One - \\
\hline & & & - Choose One - & - Chocse One - \\
\hline & & & - Choose One - & - Choose One - \\
\hline & & & - Choose One - & - Chocse One -- \\
\hline & & & - Choose One - & - Choose One -- \\
\hline & & & - Choose One - & - Choose Cne -- \\
\hline & & & - Choose One- & - Choose One - \\
\hline & & & - Choose One - & - Choose One - \\
\hline & & & - Choose One - & - Choose One -- \\
\hline
\end{tabular}


Below is an image of the front page of a "Subject Consent Form," as utilized by the IRB.

\title{
Subject Consent Form
}

Eastern Virginia Medical School (EVMS) Institutional Review Board

\author{
STUDY TITLE \\ White Light Scanner to Aid Body Contouring Surgery \\ INVESTIGATORS \\ David A. Gilbert, M.D., The Hague Center for Cosmetic Surgery \\ Robert H. Schnarrs, M.D., The Hague Center for Cosmetic Surgery \\ SPONSOR \\ The costs of this study, including administrative fees paid to the investigator(s), are being paid \\ by Novaptus Systems, Inc., who supplied the white light scanner that is being studied. Dr. \\ Gilbert is the vice-president and one of the principal equity holders in Novaptus Systems, Inc. \\ Dr. Schnarrs is also a shareholder in Novaptus Systems, Inc. Novaptus has applied for a patent \\ on the white light scanner's application to the medical industry. Dr. Gilbert and Dr. Schnarts \\ could potentially benefit from the success of the technology. \\ WHY IS THIS STUDY BEING DONE? \\ The purpose of this study is to develop digital imaging for body contouring surgery. Digital \\ imaging uses a computer to generate a three dimensional "picture" of your body contours. It is \\ our hope that this project will show that digital images may be helpful in body contouring \\ surgery.

\section{WHY ARE YOU BEING ASKED TO TAKE PART?} \\ You are being asked to participate in this research project because you are a healthy, surgical \\ candidate for body contouring surgery. \\ This is a research study. This study includes only people who choose to take part. Please take \\ your time to make your decision and feel free to ask any questions you might have. \\ WhAT ARE SOME IMPORTANT DETAILS ABOUT THIS STUDY? \\ At this local site about 500 people will take part in this study. This is a study that will take place \\ at The Hague Center for Cosmetic and Plastic Surgery. \\ We will need you to be in the study for 9 months although post-op follow-up may be extended \\ for 12 months to complete the protocol. Follow up scans will be performed at three months, six \\ months, and nine months after your body contouring surgery. \\ WHEN SHOULD YOU NOT TAKE PART? \\ If you have any of the following conditions listed below, you should not take part in this study: \\ - Pregnant \\ - Nursing \\ - History of epilepsy
}

This consent form is 8 pages long. It explains who can take part in the study and who can't. It details what is involved in the study, how many times you will get scanned, how long the scan procedure takes, and outlines the details about the scanning technology. It explains privacy issues, outlines any potential risks and benefits to those who wish to participate in the study. It discusses an "opt-out" procedure and gives explicit contact numbers should the patient have any questions or concerns.

At the end of the form is the signature block, for both the patient and a witness. There is also a signature block for the Principal Investigator.

The signature block of the Subject Consent Form is depicted below. 

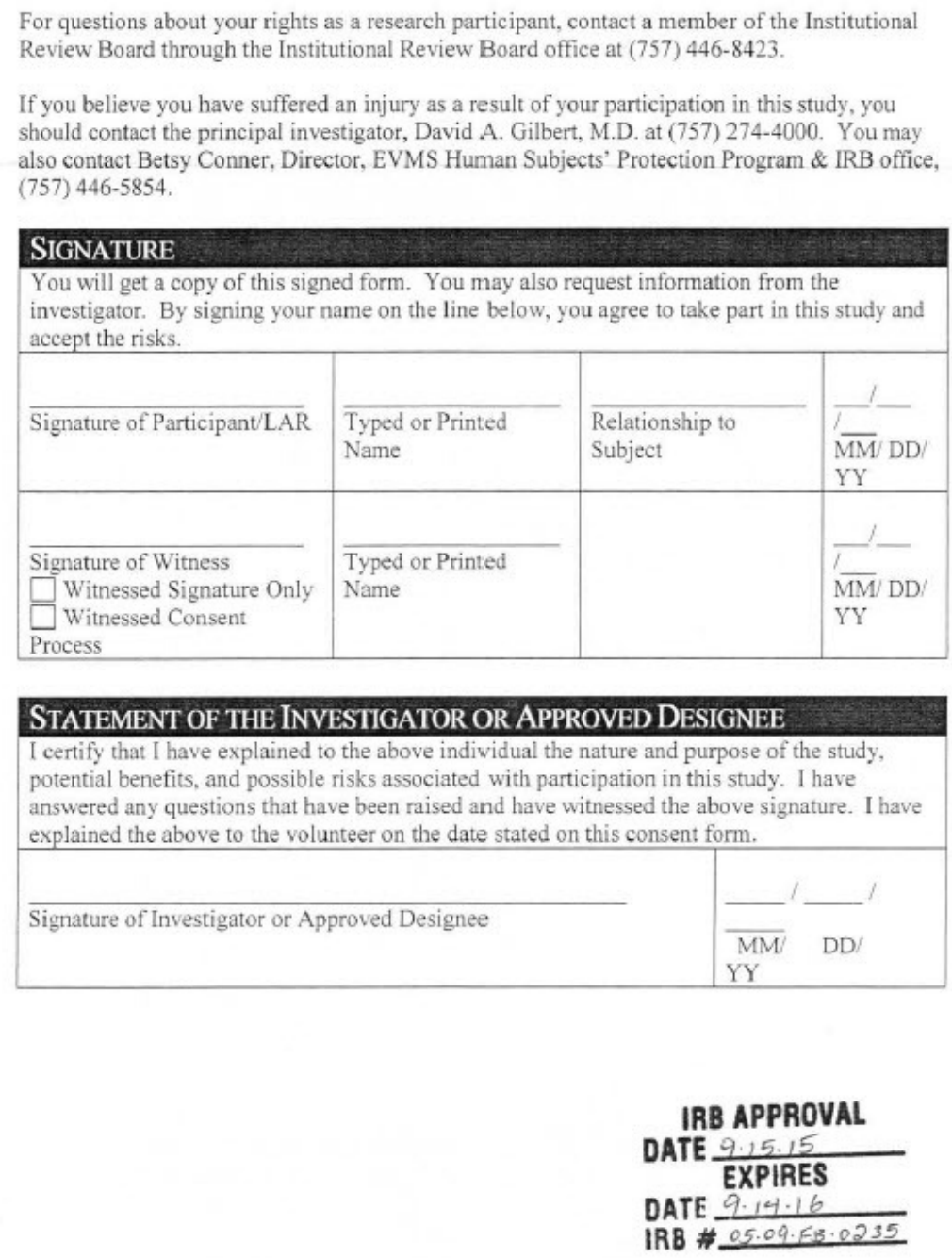

Another important point for a device considered "NSR" is that there is a prohibition on promotional activities. This is of concern if one expects to market the existing device into the medical community.

The early 3D booth-type body scanners were of such a size that they were never feasibly considered to be marketed on a mass scale. The booth-type scanner form factors have changed over the course of the years, and have come down in size quite substantially. The original size of the first 3D boothtype body scanner was 16 long by 8 feet wide and used mechanical projectors and white light stereoscopy techniques. The form factors eventually reduced down to a size of around 5 feet by 5 feet. The mechanical projectors were replaced by solid state cameras, but the white light stereoscopy technique was maintained.

The white light scanning technology became obsolete with the advent of near-field infrared scanning introduced in 2011. The sensor heads contained both the projector and camera in a convenient package that was similar to the Microsoft Kinect device used in gaming consoles. The price for these type of 3D booth-type scanners were reduced.

The present "near-field" infrared cameras commonly employed in many of the commercial 3D body scanners have of late also become on the brink of obsolescence, if they are not already obsolete.

Every time the camera technology changes, or there are other substantial changes to the device, an Amendment Assessment form must be submitted and approved by the IRB. Documentation of Substantial Equivalence should be prepared and be available for submission if requested as well.

It is the opinion of the author, however, that the rapid change in 3D scanning technology will eventually produce a small, compact, accurate, simple-to-use and portable device that will be capable of being mass-marketed at a relatively low cost, without significant changes to the camera technology. When this occurs, and is available commercially outside the medical arena, the Premarket Notification process then has a reason to commence. 


\section{Summary of How to Place a 3D Scanner into the Medical Environment for Research Purposes}

You must have a Principal Investigator who writes a protocol and submits it for approval to an accredited Institutional Review Board (IRB).

The IRB is responsible for overseeing the type of research, who can perform the research, what type of subjects can be researched, and how the data is collected and handled.

If the IRB decides that the 3D body scanner is "Non-significant risk," and approves the protocol, no IDE submission is required by the FDA and research can commence.

You must have an approved Subject Consent Form which must be explained to, and signed by, the patient prior to scanning the patient.

The research protocol is reviewed annually by the IRB.

Any significant changes to the device or the protocol's goal's must be submitted as an Amendment Assessment will be approved or rejected by the IRB at their discretion.

No active promotion of the 3D scanner in a mass commercial sense can be undertaken in that the 3D scanner utilized for research is not approved as a commercial medical device that can be marketed into the medical community.

\section{The Pre-market Notification Process}

There is a well-established process to get a medical device product into the market ${ }^{12}$. The initial step is to file a Pre-market Notification (Form 510(k)).

The following is a Pre-market flowchart as provided by the FDA:

Timeline of Communication during 510(k) Review

\section{Day 1: FDA receives $510(k)$ submission.}

\begin{tabular}{|c|}
\hline By Day 7 \\
\hline $\begin{array}{l}\text { FDA sends Acknowledgement Letter. } \\
\text { OR } \\
\text { FDA sends Hold Letter if unresolved issues with } \\
\text { User Fee and/or eCopy. }\end{array}$ \\
\hline$\downarrow$ \\
\hline By Day 15 \\
\hline $\begin{array}{l}\text { FDA conducts Acceptance Review. } \\
\text { FDA informs submitter if } 510(k) \text { is accepted for Substantive } \\
\text { Review or placed on RTA Hold. }\end{array}$ \\
\hline$\downarrow$ \\
\hline By Day 60 \\
\hline FDA conducts Substantive Review. \\
\hline $\begin{array}{l}\text { FDA communicates via a Substantive Interaction to inform } \\
\text { the submitter that the FDA will either proceed with } \\
\text { Interactive Review or that the } 510(\mathrm{k}) \text { will be placed on } \\
\text { hold and Additional Information is required. }\end{array}$ \\
\hline$\downarrow$ \\
\hline By Day 90 \\
\hline FDA sends final MDUFA Decision on $510(\mathrm{k})$. \\
\hline$\downarrow$ \\
\hline By Day 100 \\
\hline $\begin{array}{l}\text { If MDUFA Decision is not reached by Day } 100, \text { FDA provides } \\
\text { Missed MDUFA Docision Communication that } \\
\text { identifies outstanding review issues. }\end{array}$ \\
\hline
\end{tabular}

Important Notes:

- Days are Calendar Days.

- The timeline is based on the performance goals set by Medical Device User Fee Amendments of 2012 (MDUFA III).

- This timeline has been simplified. 
There are fees associated with 510K Review and the follow-up Pre-market Approval (PMA). There are also annual renewal fees which must be submitted with the appropriate documentation.

There are a series of application fees associated with the pre-market application and pre-market approval processes. Below is the fee schedule for 2016, in U.S. Dollars.

\section{FY2016 MDUFA User Fees}

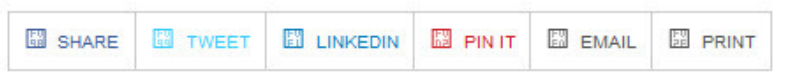

The Fees for Fiscal Year 2016 (October 1, 2015 through September 30, 2016) are as follows:

FY16 User Fees (in U.S. Dollars)

\begin{tabular}{|l|l|l|}
\hline Application Type & Standard Fee & Small Business Feet \\
\hline $510(\mathrm{~K}) \pm$ & $\$ 5,228$ & $\$ 2,614$ \\
\hline $513(\mathrm{~g})$ & $\$ 3,529$ & $\$ 1,765$ \\
\hline PMA, PDP, PMR, BLA & $\$ 261,388$ & $\$ 65,347$ \\
\hline panel-track supplement & $\$ 196,041$ & $\$ 49,010$ \\
\hline 180-day supplement & $\$ 39,208$ & $\$ 9,802$ \\
\hline real-time supplement & $\$ 18,297$ & $\$ 4,574$ \\
\hline BLA efficacy supplement & $\$ 261,388$ & $\$ 65,347$ \\
\hline PMA annual report & $\$ 9,149$ & $\$ 2,287$ \\
\hline 30-day notice & $\$ 4,182$ & $\$ 2,091$ \\
\hline
\end{tabular}

+ For small businesses with an approved SBD.

¥ Note: all types of $510(\mathrm{k})$ s (Traditional, Abbreviated, and Special) are subject to the user fee. However, there is no user fee for $510(\mathrm{k})$ s submitted to the FDA on behalf of an FDA-accredited third-party reviewer.

Small businesses with an approved SBD with gross receipts or sales of $\$ 30$ million or less are eligible to have the fee waived on their first PMA, PDP, PMR, or BLA.

Annual Establishment Registration Fee: $\$ 3,845$

There are no waivers or reductions for small establishments, businesses, or groups - all establishments must pay the establishment registration fee.

So, if you are a qualified small business, the $501 \mathrm{k}$ Pre-market Notification is $\$ 2,614$. After that, the Pre-market Approval Application is $\$ 65,347$. There is an annual Establishment Registration Fee of $\$ 3,845$. The fee list is subject to change and is updated yearly. Once published, prices are stable for that year.

\section{Summary}

We have seen that, based on historical events, self-regulation and professional conduct did not deter some from the use of questionable practices that could be considered unsafe experimentation on human subjects. These unfortunate incidents led to codification of protocols and established guidelines and policies to govern the use of research on human subjects.

The main thrust was the establishment of Independent Review Boards, informed consent, and an enforcement mechanism, generally in terms of approval of funds for continuing research. Much of this early effort was centered around internal medicine and efforts to find methods of immunization for various diseases. Drugs, in particular, now require testing for specific uses, proof of efficacy and approval by America's FDA for use within the United States, even if they were approved for specific purposes by similar agencies of other Governments.

Regulation of medical devices began in the late 1960's and was codified into law by the Medical Device Amendments of 1976. These amendments gave definition and classification to the term "Medical Device," and consolidated the approval process within America's Food and Drug Administration. 
The legislation categorized medical devices into three classes. Class I devices are considered relatively safe and have little inherent harm. These are items such as tongue depressors, surgical gloves, various types of thermometers, etc. Most are exempt from the regulatory process.

Class II are more complicated, generally require some sort of power source, require guidance in usage, and performance monitoring in action. These are generally subject to the regulatory process if the device is planned to be used commercially.

Class III devices are generally considered supportive of life functions and require the highest level of regulation.

If the device emits radiation, and technically all electronic devices emit radiation of some sort, it is subject to regulation by the FDA's Center for Device and Radiological Health. However, it can be used as an investigation device for research purposes by receiving an investigational device exemption. This does not allow the device to be commercially marketed. But it does allow the researcher to investigate the device's capabilities so long as the research protocol presented is considered a "worthy goal" and the IRB determines that usage of the device is of "Non-significant risk" to human subjects.

Further, if the IRB deems that the device used for investigation on human subjects to be of "Nonsignificant Risk," it is exempt from the requirement to submit an IDE approval to the FDA.

This is where current 3D body scanners, including booth-type scanners find themselves at present for use within a medical environment. Though they are used extensively in the clothing world, and even in the fitness arena, direct usage in a medical environment requires an investigation path, and that path is through finding a sponsor as a qualified "Principal Investigator," an accredited Institutional Review Board that determines that the research goals are worthy, and that the 3D scanner can be classified as "NSR."

In addition to the defined research goals and the "NSR" designation, consent forms must be utilized for all human subjects on which the device will be used. Further, an annual review of the research study is required, and there is often a fee associated with the IRB, though this depends on the institution.

This allows the investigator to obtain data for analysis, and to prove the merit of using the multidimensional measurement capabilities provided by the 3D scanner that were presently unavailable.

And this is where we stand today, in October of 2016.

The 3D booth-type scanner has shown value in both cosmetic and bariatric surgery arenas. But the rapid rate of technological change in scanner sensor technology has made it prohibitive and uneconomical to begin the Pre-market 501k submission process that would lead to a pre-market approval (PMA) authorization.

In fact, simply changing scanner models from the original and now obsolete white light sensors to the near-obsolete near-field infrared sensors requires an amendment to the IRB protocol. It is still considered to be "NSR," but the modification process takes time and documentation.

\section{Conclusions}

It is possible to present a $3 D$ body scanner in such a way as to have it classified with an "Investigational Device Exemption," allowing it to be used for research purposes on human subjects. Further, the IDE designation is not required if the IRB deems the device to be of a "Non-significant risk) nature. The path taken to do this has been outlined above.

This only goes so far. Once the research has yielded data that can be positively analyzed against the research goals, the next step must be taken.

That step is to begin the formal Pre-market certification process to get the body scanner classified as a Class II Medical Device. The requirements themselves for this classification of equipment are not too onerous, though there are multiple fees to be paid, both for submission of the Pre-market application (510k) and the fee for the Pre-Market Approval (PMA) Application. There is an annual renewal fee and supporting documentation that must be submitted. 
The reason that this next step has not been taken yet is the rapid advancement in scanning technology. This rapid advancement tends to obsolete existing scanner sensor head technology. This is why the focus has been on developing software and algorithms that are universal in nature and not dependent on a particular scanner model or manufacturer.

The day will come when a 3D whole-body scanner will appear in the commercial market that will be more economical, easy to use and much smaller and less costly to manufacture. It may even be portable in nature and calibrate itself quickly and easily. This will most likely happen in the commercial space, outside the medical community. Inside the medical environment, it needs to be located near the stadiometer in a general practitioner's office. Perhaps it will be an attachment to the standard stadiometer that exists in practices all over the world today. It could even be in the form of a "smartmirror" on a wall next to the stadiometer.

This type of scanner would then have the potential to become as commonplace as a stethoscope or blood pressure cuff in a generalized primary care practice. Once a 3D whole body scanner of this type is identified, the IRB process, followed correctly and studiously, will allow it to be evaluated and compared to the data already collected from 3D booth-type body scanners that have been utilized to date in medical practices for research purposes.

At that point, it certainly makes sense to begin the Pre-market $501 \mathrm{~K}$ procedure and follow through with the entire commercialization process needed for mass distribution. There should be adequate use in the commercial world that would allow enough information to convince the FDA that usage of this type of scanner in the medical world is "substantially equivalent" to its usage in the commercial world.

At that point, 3D surface body scanning in the medical community will become as common as a stethoscope.

\section{References}

1. http://ors.umkc.edu/research-compliance-(iacuc-ibc-irb-rsc)/institutional-review-board-(irb)/history-ofresearch-ethics

This is a good summary of the incidents that led up to the rules and regulations for medical research on human subjects that we have today. Most other articles have similar content.

2. http://www.iupui.edu/ histwhs/G504.dir/irbhist.html

This is an excellent summary of the history of medical ethics as applied to research on human subjects. There are several links within the summary that offer additional background, including links to the Prussian doctor mentioned, as well as numerous links on the background of the Nuremburg trials and the Nuremburg Code.

3. https://helix.northwestern.edu/article/thalidomide-tragedy-lessons-drug-safety-and-regulation A condensed article on the Thalidomide Tragedy and the resulting U.S. Senate hearings.

4. http://www.cdc.gov/tuskegee/timeline.htm The Centers for Disease Control article on the Tuskegee Syphilis Study.

5. http://www.hhs.gov/ohrp/regulations-and-policy/belmont-report/index.html The Health and Human Services site for the original Belmont Report.

6. http://www.fda.gov/AboutFDA/CentersOffices/OfficeofMedicalProductsandTobacco/CDRH/CDRHTranspare ncy/ucm203018.htm

7. http://www.accessdata.fda.gov/scripts/cdrh/cfdocs/cfcfr/CFRSearch.cfm?CFRPart=1000\&showFR=1\&subpa rtNode $=21: 8 \cdot 0 \cdot 1 \cdot 3 \cdot 37.1$

8. http://www.accessdata.fda.gov/scripts/cdrh/cfdocs/cfcfr/CFRSearch.cfm?CFRPart=1000\&showFR=1\&subpa rtNode $=21: 8 \cdot 0 \cdot 1 \cdot 3 \cdot 37.2$

9. http://www.fda.gov/downloads/medicaldevices/newsevents/workshopsconferences/ucm499250.pdf

10. http://www.fda.gov/downloads/medicaldevices/deviceregulationandquidance/guidancedocuments/ucm2791 $\underline{07 . p d f}$

11. https://tracs.unc.edu/index.php/services/regulatory/ind-and-ide-application-support/investigational-deviceexemptions

12. http://www.fda.gov/medicaldevices/deviceregulationandguidance/howtomarketyourdevice/premarketsubmiss ions/premarketnotification510k/default.htm

13. http://www.fda.gov/MedicalDevices/DeviceRegulationandGuidance/HowtoMarketYourDevice/PremarketSub missions/PremarketNotification510k/ucm070201.htm

14. http://www.fda.gov/ForIndustry/UserFees/MedicalDeviceUserFee/ucm452519.htm 